\title{
La commercializzazione del vino e dell'olio in Italia settentrionale attraverso lo studio dei daziari tardomedievali
}

\author{
di Riccardo Rao
}

Il contributo ricostruisce il commercio del vino e dell'olio in Italia settentrionale a partire dall'analisi dei tariffari tardomedievali. I tariffari delle città maggiori sono caratterizzati da un'ampia gamma di prodotti vinicoli, che includeva i vini greci, ma anche vini commerciati a livello regionale. L'olio di oliva insieme all'olio di lino costituisce la varietà più importante fra gli oli da cucina.

The contribution reconstructs the wine and oil trade in Northern Italy, starting from the analysis of late medieval tariff books. The tariff books of the major cities are characterised by a wide range of wine products, which included Greek wines, but also wines traded regionally. Olive oil together with linseed oil constitutes the most important variety among cooking oils.

Medioevo; secoli XIII-XV; Italia settentrionale; olio; vino; commercializzazione; tariffari.

Middle Ages; $13^{\text {th }}-15^{\text {th }}$ centuries; Northern Italy; oil; wine; commecialization; tariff books.

Questa ricerca è un prodotto del PRIN 2017 Loc-Glob. The local connectivity in an age of global intensification, con la partecipazione delle Unità di Bergamo (coordinatore scientifico e responsabile di ricerca: Riccardo Rao), Sassari (responsabile unità di ricerca: Pinuccia Simbula); Torino (responsabile unità di ricerca: Enrico Basso) e Udine (responsabile unità di ricerca: Bruno Figliuolo).

Riccardo Rao, La commercializzazione del vino e dell'olio in Italia settentrionale attraverso lo studio dei daziari tardomedievali, pp. 275-284, () 2021 Author(s), content CC BY 4.0 International, DOI 10.36253/97888-5518-423-6.16, in Paola Guglielmotti, Isabella Lazzarini (edited by), «Fiere vicende dell'età di mezzo». Studi per Gian Maria Varanini, (c) 2021 Author(s), content CC BY 4.0 International, metadata CC0 1.0 Universal, published by Firenze University Press (www.fupress.com), ISSN 2704-6079 (online), ISBN 97888-5518-423-6 (PDF), DOI 10.36253/978-88-5518-423-6 
Per chi voglia oggi affrontare alcuni rilevanti temi di storia agraria, di notevole fortuna soprattutto negli anni Ottanta e Novanta del Novecento, quali la storia del vino e quella dell'olivocoltura in Italia, è indispensabile leggere i contributi sull'argomento di Gian Maria Varanini, che si caratterizzano per il prestare ampio spazio, ancor più che alle tecniche colturali e agli aspetti paesaggistici, alle dinamiche di commercializzazione di tali prodotti. Portando alla luce i complessi circuiti regionali che regolavano la distribuzione di vino e olio, Varanini ha superato i quadri ancora a volo d'uccello, con una marcata deformazione prospettica per il commercio internazionale, suggeriti dal Melis ${ }^{1}$.

A partire da tali intuizioni, in questa sede si intende tornare sul tema della commercializzazione di vino e olio in Italia settentrionale, proponendo un esame comparativo delle fonti daziarie. Tali fonti, ora al centro di un Progetto di Rilevante Interesse Nazionale (PRIN 2017) Loc-Glob, centrato sulla connettività locale nel tardo medioevo, costituiscono infatti un osservatorio privilegiato, tutto sommato relativamente poco sfruttato, per ricostruire i circuiti commerciali e l'integrazione economica delle produzioni agricole ${ }^{2}$. In particolare, si è deciso di concentrare l'attenzione su un primo limitato corpus di testi proveniente dall'Italia settentrionale, relativo ai daziari di Rivoli (1268), Mondovì (1276), Ivrea (1313), Venzone e Chiusaforte (1315), Como (1342ca e 1400-1450ca), Morbegno (1342), Udine (1363), Bellinzona (1375ca, 1389), Riva del Garda (1370), Piacenza (1380), Parma (1386), Pavia (1365, 1383 e 1400), Verona (1409), Brescia (1427-1428), Bergamo (1431), Melegnano (1343-1416), Milano (1317, 1346, 1365, 1450 circa), Masino (1450), Novara (1475 secolo) $)^{3}$.

\footnotetext{
${ }^{1}$ In particolare, si fa riferimento a Varanini, Le strade del vino e a Varanini, L'olivicoltura e l'olio gardesano. Per gli studi di Melis su olio e vino si rimanda almeno a Melis, I vini italiani nel Medioevo e a Melis, Note sulle vicende storiche dei commerci oleari.

${ }^{2}$ Per il vino in area piemontese tali fonti sono state valorizzate da Greci, Il commercio del vino negli statuti comunali, pp. 251-254.

3 Tra gli studiosi che stanno partecipando all'alimentazione del GIS che raccoglie i tariffari dell'Italia tardomedievale, desidero ringraziare Paolo Buffo, Alessia Meneghin e Tommaso Vidal per la condivisione delle informazioni. Si riportano qui i riferimenti archivistici e bibliografici dei daziari usati nel testo. Per Rivoli, Mondovì e Masino: Daviso di Charvensod, I pedaggi delle Alpi occidentali, pp. 316-317; 423-424, 459-461. Per Ivrea: Buffo, I documenti dell'Archivio storico del comune di Ivrea, doc. 55, pp. 294-302. Per Venzone e Chiusaforte: Archivio di Stato di Venezia, Commemoriali, reg. I, c. 232r. Per Como e Bellinzona, gli statuti sono editi, da un codice di Bellinzona, da Liebenau, Le ordinazioni daziarie di Como nel XIV secolo: ulteriori disposizioni in originale sono conservate in Archivio di Stato di Como, Datii civitatis Novocomi de anno 1335 ad annum 1350, e consentono di cogliere le integrazioni e la stratificazione cronologica. Per Milano 1450: Liber datii mercantie communis Mediolani. Per Morbegno: Archivio di Stato di Sondrio, Atti dei Notai, m. 2, c. 319r. Per Udine: Biblioteca civica di Udine, Fondo Principale, ms. 841 (Ordinamenta daciorum et statuta comunitatis Utini). Per Riva del Garda: Archivio storico del comune di Riva del Garda, Libro Giornale n. 25, ff. 2-3. Per ragioni di reperibilità in tempi di Covid non mi è stato possibile consultare integralmente il daziario di Piacenza edito da Castignoli, Liber daciorum et officiorum communis Placentie: i riferimenti a tale testo saranno pertanto non sistematici. Per Parma: Biblioteca Palatina di Parma, Manoscritto Parm. 533, f. 45. Per Pavia: Biblioteca Civica Bonetta, Archivio storico civico di Pavia, Parte antica, Dazi alle porte, A II 9, individuato da Fabio Romanoni, che ringrazio per la segnalazione
} 


\section{Le strade dei vini e i liquori}

Il commercio dei vini nell'Italia tardomedievale, che conta ormai su una solida letteratura scientifica, è noto nei suoi tratti essenziali ${ }^{4}$. È stato già messo in evidenza il forte afflusso di vini d'importazione dall'Egeo, reso possibile dall'abbattimento dei costi di trasporto fra Tre e Quattrocento: i vini di Creta, di Rodi e Romanìa sono tra i principali protagonisti dei consumi enologici in questo periodo ${ }^{5}$. Ma è stata anche sottolineata l'esistenza di mercati vitivinicoli di ambito regionale, così come la relazione stretta tra le città e il loro territorio ${ }^{6}$.

Tali dinamiche di importazione del vino sono verificabili anche dalle pratiche di mercatura, che menzionano spesso i vini provenienti dall'Oriente ${ }^{7}$. Tuttavia, i daziari restituiscono in maniera molto più articolata una simile complessità, delineando il doppio binario di importazione internazionale e di circuiti regionali: quelli dei centri maggiori, come Milano, Novara, Pavia, Brescia e Bergamo, ma anche Udine, menzionano il vino, la malvasia e il moscatello di Creta, nonché i vini di Rodi, di Tiro della Romanìa. Al contrario, i vini "navigati" sono assenti nei daziari centri minori, a segno che la loro presenza doveva essere trascurabile. Mancano in quelli di Latisana (1261), Ivrea (1312), Rivoli (1286) e Morbegno (1342), Melegnano (1416) e Masino (1450), che indicano il dazio in maniera generica per ogni tipo di vino (indizio forse di uno smercio soprattutto di produzioni locali) ${ }^{8}$.

Tuttavia, i vini greci non sono menzionati neppure nei daziari milanesi precedenti alla metà circa del Trecento: quello di Milano (1317), per esempio, tra le merci per uso alimentare importate da Venezia menziona solo le spezie. Quello di Parma del 1386, invece, destina un unico dazio ai vini «tanto puri quanto mescolati». Non fanno distinzioni specifiche sulla qualità dei vini neppure i daziari di Como, né quello di metà Trecento, né quello del secolo successivo. Tali dati suggeriscono che i vini d'Oriente, seppur reperibili, si siano

e che contiene anche i daziari di Milano fra il 1346 e il 1365; Biblioteca Universitaria di Pavia, ms. Aldini, ms. 506, Libro dei dazi di Milano e di Pavia (secc. XIV-XV). Per Verona: Archivio di Stato di Verona, Archivio antico del comune di Verona, b. 499 (1388); Camera fiscale, b. 116 (1409). Per Brescia: Albini, La tariffa del dazio della mercanzia a Brescia. Per Bergamo: Biblioteca civica Angelo Mai, Contractus datiorum Bergomi, Brixiae 1575. Per Novara: Statuta civitatis Novariae.

4 Rimando innanzitutto al contributo fondamentale, anche per problematizzazione storiografica, di Varanini, Le strade del vino, che aggiorna il fondamentale contributo storiografico di Pini, Medioevo nel bicchiere.

5 Melis, I vini italiani nel Medioevo, p. 178; Pini, Il vino del ricco e il vino del povero.

6 Varanini, Le strade del vino; Balestracci, Il consumo del vino; Greci, Il commercio del vino negli statuti comunali. Per le tipologie di vini presenti nell'Italia tardomedievale si veda anche Gaulin, Tipologia e qualità dei vini.

7 Per esempio, si veda Pegolotti, La pratica della mercatura, p. 39, che menziona il vino greco proveniente da Costantinopoli.

8 Converge con tale osservazione l'esame delle fonti tariffarie del Piemonte occidentale da parte di Greci, Il commercio del vino negli statuti comunali, pp. 251-254. 
affermati solo progressivamente con flussi continui nel corso del Trecento, soprattutto nelle aree meglio commercializzate della Lombardia.

Rispetto a questi prodotti internazionali che sono presenti in maniera ormai diffusa nell'intera Penisola, altri vini circolano invece all'interno di un orizzonte di ambito regionale. Esistono cioè vini che riescono a varcare le frontiere dei contadi di produzione, per riversarsi sui centri urbani contermini: in tal modo da "vini nostrani", così come sono indicati per lo più nei daziari, assumono una più chiara identificabilità onomastica ${ }^{9}$. Per esempio, nell'area lombardo-viscontea, il vino di Pavia (vinum Papiense), prodotto nel distretto di tale città (dunque non solo nella zona vocazionale dell'Oltrepò, ma anche nelle campagne pianeggianti del Pavese e della Lomellina), era smerciato sui mercati di Pavia e Milano. Più pregiato doveva essere il vino di Valtellina (vinum Oltrinascum), che, oltre a essere venduto Oltralpe, nel dominio visconteo è presente nei daziari di Milano, Pavia e Novara. I tariffari comaschi, che contenevano un'apposita legislazione statutaria sul commercio e l'esportazione del vino, a dimostrazione dell'importanza della produzione del territorio, rilevano le maggiorazioni imposte al vino che veniva esportato Oltralpe, pur senza indicarne la qualità ${ }^{10}$. Anche il daziario di Bellinzona conferma il passaggio di vini rossi del versante italico verso Oltralpe ${ }^{11}$.

Si può provare a capire l'evoluzione del mercato del vino tardomedievale attraverso il confronto dei daziari tre e quattrocenteschi di Milano. La normativa in materia di tariffe prodotta dai Visconti attorno al 1346 individuava nel dettaglio i vini importati. Dall'Egeo proveniva la malvasia di Creta. Vini pregiati d'importazione erano anche la vernaccia, verosimilmente attraverso la Liguria, e la Ribolla friulana o istriana. Segue una serie di vini che mostrano la capacità di Milano di drenare le produzioni enologiche provenienti dai territori circostanti, che iniziano a disegnare un ampio circuito regionale ${ }^{12}$. Innanzitutto, quelli confinanti con il Milanese, come il vino della Gera d'Adda e della Riviera dell'Adda, del Bergamasco, del Novarese, di Romagnano Sesia (forse più pregiato), ma anche il vino valtellinese, il moscatello pavese, probabilmente una produzione imitativa dei vini egei, fino ai vini di Lombardia in generale e della Marca (trevigiana, probabilmente). La varietà di vini reperibili a Milano a metà Quattrocento appare ancora maggiore, anche rispetto ai coevi daziari di Novara e a Pavia. Compaiono ancora i vini "navigati" importati da Venezia (vini di Creta e di Rodi, malvasia e moscatello), insieme a quel-

9 Come osserva Montanari, è nel momento in cui i prodotti alimentari escono dal territorio di riferimento che iniziano a essere riconoscibili: Capatti, Montanari, La cucina italiana, pp. VIII-IX.

${ }^{10}$ Liebenau, Le ordinazioni daziarie di Como, pp. 277-282; Archivio di Stato di Como, Datii civitatis Novocomi de anno 1335 ad annum 135o, ff. 18r, 34r, 48r.

${ }^{11}$ Liebenau, Le ordinazioni daziarie di Como, p. 292; Archivio di Stato di Como, Datii civitatis Novocomi de anno 1335 ad annum 135o, f. 24 r.

${ }^{12}$ Sull'emersione di vitigni ancor oggi identificabili nelle differenti aree regionali della penisola si veda, per il Piemonte del tardo medioevo, Nada Patrone, Il consumo del vino nella società pedemontana, pp. 288-292. 
li locali (nostrano) e ai principali di orizzonte regionale (pavese e valtellinese). Si potevano poi acquistare i vini provenienti da Genova, come la vernaccia e il trebbiano (probabilmente di produzione toscana), non menzionato nel Trecento, la ribolla, il vino della Marca e i vini, non identificati da questa ricerca, «de la Qualea» e «de Maraile». Per contro non trova spazio nei daziari quattrocenteschi l'importazione di vino bergamasco, che pure era stata oggetto di specifici interventi da parte dei Visconti nel XIV secolo ${ }^{13}$.

In area veneta, invece, i vini di riferimento per il mercato regionale sono diversi. Il vino di Slavonia è quasi un marcatore per lo Stato di Terraferma: la sua presenza dal Friuli (dove il vino di Slavonia è anche indicato come d'Oltre Livenza) arriva fino a Verona, dove veniva smerciato anche l'aceto proveniente da quella zona (il dazio era di un terzo superiore a quello imposto all'aceto comune), mentre non compare in alcun daziario lombardo tra quelli presi in esame. Quasi sempre presenti sono naturalmente anche la ribolla e il vino della Marca trevigiana, che tuttavia venivano ampiamente smerciati anche al di fuori della terraferma veneta. Il daziario di Udine registra una varietà di produzioni regionali particolarmente articolata, che imita anche i prodotti internazionali più ricercati: oltre alla ribolla (sia di Collio, sia istriana), compare infatti il moscatello istriano. Figurano poi il vino della Marca trevigiana, il vino di Montello e il trebbiano, che pure è solitamente ritenuto un prodotto trattato in prevalenza dai Genovesi. A Verona, invece, la generica voce «vini forestieri» limita la possibilità di verificare nel dettaglio la circolazione vinicola su tale piazza (anche se, come si è detto, alcuni prodotti internazionali e regionali particolarmente ricercati sono menzionati con una voce specifica): è significativo che, tra i vini locali, si distingua invece tra il «vinum de Monte» e quello «de Plano», il secondo dei quali daziato per un valore di due terzi inferiore, che suggerisce già un chiaro apprezzamento dei vini delle valli veronesi.

Prodotti all'indomani dell'ingresso nello Stato di Terraferma, i daziari di Bergamo e Brescia non denotano invece una chiara identità regionale, né di area veneta, né lombarda. A Bergamo, come beni d’importazione sono presenti soltanto i classici vini navigati da Venezia, come la malvasia e i vini di Tiro e di Romanìa, ma anche la vernaccia. Potrebbe essere una produzione locale, come vedremo tra poco, anche il moscatello, che ha un dazio elevato (10 lire il carro), ma decisamente inferiore rispetto ai vini d'importazione (tutti a 15 lire al carro). Compare inoltre come prodotto di qualità un generico vinum album (vino bianco), più pesantemente daziato rispetto ai vini citrini (paglierini) e vermilii (rossi): 8 lire al carro per il primo contro 7 per i secondi. Sono infine incluse numerose norme sulla commercializzazione del vino che tuttavia, senza alludere a vini di circolazione regionale, lasciano intendere di riferirsi soprattutto alle produzioni locali.

\footnotetext{
13 Per i vini di importazione su cui si erano diversamente specializzate Genova e Venezia si rimanda a Pini, Il vino del ricco e il vino del povero, pp. 590-595.
} 
A Brescia, invece, i principali vini di importazione erano la vernaccia, proveniente da Genova (vernacia Ianuensis), la ribolla, la malvasia e i vini di Creta e di Romanì ${ }^{14}$. Dal vicino territorio bergamasco proveniva inoltre il moscatello - ancora oggi una produzione caratteristica per via del moscato di Scanzo - di cui fin d'allora era pertanto stata avviata una produzione imitativa dei più ricercati prodotti d'Oltremare: è del resto suggestivo che ancora oggi le due aree lombarde di produzione di moscato siano proprio la Bergamasca e il Pavese, dove, come si è visto, tali indirizzi erano stati intrapresi fra Tre e Quattrocento ${ }^{15}$.

Infine, i daziari menzionano la presenza di liquori. Il vino di melograno e l'acquavite potevano essere, infatti, acquistati a Milano, Pavia e Novara: il fatto che siano indicati come spezie potrebbe suggerire che si trattasse di prodotti d'importazione ${ }^{16}$. Non sorprende neppure di ritrovare, nei daziari di Milano, lo zibibbo, che anche le fonti cronachistiche lombarde menzionano come un liquore particolarmente pregiato sino almeno dalla fine del Trecento ${ }^{17}$.

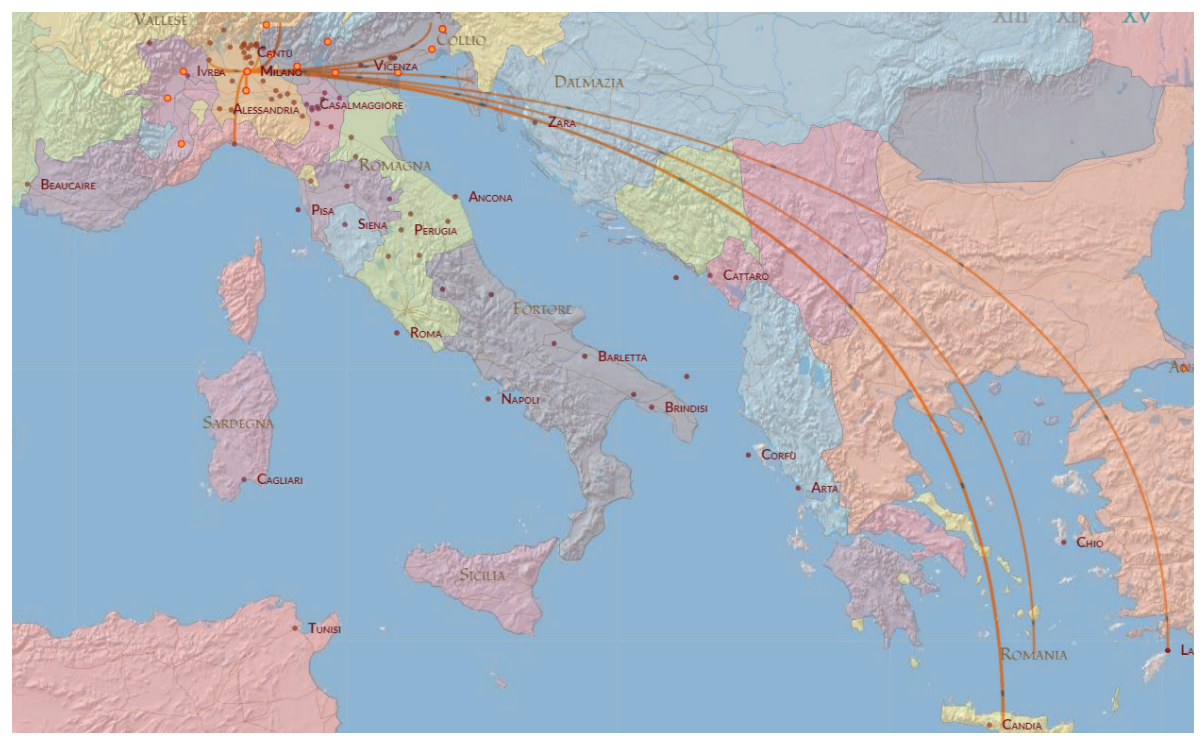

Carta 1. Le aree di importazione del vino a Milano secondo i daziari tardomedievali (tratte dal GIS Loc-Glob).

\footnotetext{
${ }^{14}$ Sulla vernaccia e sui vini liguri si vedano anche le osservazioni di Figliuolo, Giuliani, Merci e mercanti e di Melis, I vini italiani nel Medioevo, p. 62.

${ }_{15}$ Per le produzioni imitative: Pini, Il vino del ricco e il vino del povero, pp. 594-595. La produzione di moscatello in alcune aree del Piemonte meridionale è messa in evidenza da Nada $\mathrm{Pa}$ trone, Il consumo del vino nella società pedemontana, pp. 288-289. Invita comunque prudenza nello stabilire eccessivi tratti di continuità tra le produzioni tardomedievali e quelle attuali, verificandone anche le consistenti discontinuità, Grieco, I sapori del vino, pp. 166-167.

16 Per il consumo di acquavite, documentato sin dal Duecento, si veda Sandri, Grieco, Appunti per una storia dell'acquavite.

${ }_{17}$ Montanari, La fame e l'abbondanza, p. 92, ne ritrova menzione nella cronaca del piacentino Giovanni de Mussis.
} 


\section{Aspetti della commercializzazione dell'olio nei daziari}

Già i trattati di mercatura mettono in evidenza l'emersione di alcune grandi aree di produzione dell'olio di oliva tardomedievale. Per esempio, il Pegolotti menziona, tra gli oli italici che si potevano acquistare sulle maggiori piazze della penisola e del Mediterraneo, quelli delle Marche, della Puglia e della Campania ${ }^{18}$. Al pari del vino, a fianco di questi oli di distribuzione “internazionale", ne esistevano altri dai circuiti decisamente più regionali. Gian Maria Varanini ha osservato che, sebbene l'olio di oliva costituisca per lo più nell'Italia centro-settentrionale un bene il cui consumo era ristretto alle élites, nelle aree di produzione, come la zona del Garda, poteva essere diffuso fin negli strati più umili della popolazione ${ }^{19}$. Il daziario di Verona del 1409 conferma senz'altro una simile intuizione, menzionando anche l'oleum Brixiense, a indizio che la produzione del territorio gardesano di sponda veronese non era sufficiente ad appagare la richiesta del mercato di olio di oliva, che veniva anche importato dalla riva bresciana ${ }^{20}$. Osservazioni simili possono essere estese alla stessa Brescia, pure interessata dalla produzione gardesana. Il daziario del 1431 dedica diversi capitoli al commercio di olio di oliva e di olive verdi, regolandone l'esportazione e lasciandone intendere una diffusa produzione per l'autoconsumo ${ }^{21}$. Il fatto che si alluda all'importazione dell'oleum Marchianum, da identificare con tutta probabilità con il ben documentato olio marchigiano importato attraverso Venezia, sembra suggerire una richiesta urbana che non si accontentava dell'offerta garantita dalla produzione locale ${ }^{22}$.

Anche a Como la produzione olearia era sviluppata: in questo caso, tanto nei daziari trecenteschi, quanto in quelli quattrocenteschi, l'olio era smerciato anche Oltralpe, dove subiva una tassazione aggiuntiva rispetto alle altre esportazioni. Peraltro, l'olio di oliva è l'unico tipo di olio daziato nella città lariana $^{23}$. Non manca l'olio d'oliva in entrata e in uscita neppure nel trecentesco

\footnotetext{
18 Pegolotti, La pratica della mercatura, pp. 39, 109. In generale, sul commercio dell'olio si veda almeno Melis, Note sulle vicende storiche dei commerci oleari, tuttavia limitato agli aspetti più generali delle produzioni mediterranee e ora in buona misura superato anche dalla conoscenza più puntuale delle produzioni dei territori locali. Fondamentale il recente Ars olearia, con particolare riferimento ai contributi di Basso, L'olio sul mare, e Orlandi, L'olivo e l'olio tra Mediterraneo e Mare del Nord.

19 Varanini, L'olivicoltura e l'olio gardesano, p. 178.

${ }^{20}$ Per l'ampia commercializzazione dell'olio gardesano, con anche l'analisi dei dazi sull'olio a Verona, si rimanda a Varanini, L'olivicoltura e l'olio gardesano, pp. 168-183.

${ }^{21}$ Albini, La tariffa del dazio della mercanzia a Brescia, p. 8 (suggerisce l'autoconsumo anche «quilibet civis portari facere possit extra civitatem pro suo uxu usque ad unam baxetam sine datio»).

${ }^{22}$ Per il rilevante ruolo commerciale svolto nel tardo medioevo dall'olio delle Marche, importato attraverso Venezia, si vedano anche Brugnoli, Varanini, Introduzione. Olivi e olio, pp. 6o-61; Basso, L'olio sul mare, p. 85.

${ }^{23}$ Liebenau, Le ordinazioni daziarie di Como, p. 219; Archivio di Stato di Como, Datii civitatis Novocomi de anno 1335 ad annum 135o, ff. 11r, $29 r$.
} 
daziario di Riva del Garda, dove probabilmente venivano intercettati i carichi di olio diretti a nord ${ }^{24}$.

Ad ogni modo, l'olio di oliva si presenta ormai in molte città dell'Italia settentrionale come l'olio commestibile per eccellenza. A Parma, per esempio, il daziario del 1386 lo distingue dagli altri oli, che sono invece classificati come a comburendo, dunque usati essenzialmente come combustibile. Tuttavia, a ben vedere, nella maggior parte delle grandi città lombarde ed emiliane, gli oli destinati al consumo alimentare sono quasi sempre due: i daziari di Milano, Pavia, Novara e persino delle città lacustri di Brescia e Verona affiancano infatti all'olio di oliva quello di lino. A Pavia, compare anche quello di noci, espressamente segnalato, così come quello di lino, come una produzione nostrana $^{25}$. A Ivrea, invece, nel 1313 a una voce generica per l'olio (forse di oliva e anche di lino) se ne affianca una specifica per quello di noci.

Risulta invece più difficile comprendere il consumo degli oli alimentari nei centri minori. I daziari di queste località - per esempio quelli di Latisana, Venzone, Chiusaforte, Mondovì e Masino (ma l'osservazione riguarda anche i tariffari di alcuni centri maggiori, come Piacenza, che menzionano semplicemente l'«oleum ad comendum conductum in civitate») - spesso destinano infatti una voce generica all'olio, in cui probabilmente non si faceva distinzione tra quello di oliva e gli altri.

Insomma, a una vista d'insieme, gli oli alimentari dominanti nel consumo sembrano essere quello di oliva e quello di lino, la cui conoscenza nella letteratura scientifica mi sembra nel complesso piuttosto limitata, mentre il ben noto olio di noci, pur non essendo assente, sembra avere una diffusione più contenuta, maggiormente marcata soltanto in area piemontese ${ }^{26} . \mathrm{Nel}$ complesso, dai daziari emerge una presenza non del tutto trascurabile degli oli usati a fini alimentari, almeno per gli ambiti urbani e più commercializzati, in particolare di quelli di oliva e di lino, che contribuisce a una maggiore comprensione dell'uso in cucina di questi grassi ${ }^{27}$.

\footnotetext{
${ }^{24}$ Archivio storico del comune di Riva del Garda, Libro Giornale n. 25, f. $3 v$ : edizione in Regesti documenti capsa III e IV - Comune di Riva del Garda, p. 249.

25 Biblioteca Civica Bonetta, Archivio storico civico di Pavia, Parte antica, Dazi alle porte, A II 9, 1382: «oleum nucum et linose factum in episcopatu Papie».

${ }_{26}$ Per esempio, individua l'olio di noci come dominante nei consumi lombardi e piemontesi Naso, Usi alimentari, pp. 425-429. Per un inquadramento dell'uso di materie grasse e oli in Europa si veda Montanari, Tradizioni regionali e modelli culinari.

${ }^{27}$ Grieco osserva un uso limitato dell'olio a fini alimentari nell'Italia tardomedievale, che supera gli stereotipi sulla cucina mediterranea: Grieco, Olive tree cultivation and the alimentary use of olive. Il ruolo dell'olio nell'alimentazione è sinteticamente inquadrato anche da Brugnoli, Varanini, Introduzione. Olivi e olio, pp. 68-81.
} 


\section{Opere citate}

G. Albini, La tariffa del dazio della mercanzia a Brescia nel sec. $X V$, in «Libri\&Documenti», 4 (1978), 3, pp. 1-19.

Ars olearia. Dall'oliveto al mercato nel medioevo - Ars olearia. From Olive Grove to Market in the Middle Ages, a cura di I. Naso, Guarene 2018.

D. Balestracci, Il consumo di vino nella Toscana bassomedievale, in Il vino nell'economia e nella società italiana medioevale e moderna, Atti del Convegno (Greve in Chianti, 21-24 maggio 1987), Firenze 1988, pp. 13-29.

E. Basso, Lolio sul mare. Il commercio oleario nel basso medioevo, in Ars olearia, pp. 79-105.

A. Brugnoli, G.M. Varanini, Introduzione. Olivi e olio nel medioevo italiano, in Olivi e olio nel medioevo italiano, pp. 3-100.

P. Buffo, I documenti dell'Archivio storico del comune di Ivrea, in «Bollettino storico-bibliografico subalpino», 100 (2012), 1, pp. 201-308.

L. Capatti, M. Montanari, La cucina italiana. Storia di una cultura, Roma-Bari 2005.

P. Castignoli, Liber daciorum et officiorum communis Placentie (anno MCCCLXXX). L'appalto delle gabelle e degli uffici in un comune cittadino del dominio visconteo, Roma 1975.

La civiltà del vino. Fonti, temi e produzioni vitivinicole dal Medioevo al Novecento, Atti del convegno (Monticelli Brusati - Antica Fratta, 5-6 ottobre 2001), a cura di G. Archetti, Brescia 2003.

Dalla vite al vino. Fonti e problemi della vitivinicoltura italiana medievale, a cura di J.-L. Gaulin, A.J. Grieco, Bologna 1994.

M.C. Daviso di Charvensod, I pedaggi delle Alpi occidentali nel medioevo, Torino 1961.

B. Figliuolo, A. Giuliani, Merci e mercanti pisani a Firenze e fiorentini a Pisa nei registri doganali trecenteschi, Roma 2020.

J.-L. Gaulin, Tipologia e qualità dei vini in alcuni trattati di agronomia italiana (sec. XIV-XVII), in Dalla vite al vino, pp. 59-83.

R. Greci, Il commercio del vino negli statuti comunali di area piemontese, in Vigne e vini nel Piemonte medievale, pp. 245-280.

A.J. Grieco, I sapori del vino: gusti e criteri di scelta tra Tre e Cinquecento, in Dalla vite al vino, pp. 165-186.

A.J. Grieco, Olive Tree Cultivation and the Alimentary Use of Olive Oil in Late Medieval Italy (ca. 1300-150o), in La production du vin et de l'huile en Mediterranée, a cura di M.C. Amouretti, J.P. Burn, Athènes 1993, pp. 297-306.

Liber datii mercantie communis Mediolani. Registro del secolo $\mathrm{XV}$, a cura di A. Noto, Milano 1950.

T. Liebenau, Le ordinazioni daziarie di Como nel XIV secolo, in «Periodico della Società storica comense», 17 (1885), pp. 204-294.

F. Melis, Note sulle vicende storiche dei commerci oleari (secoli XIV-XVI), in Olivi e olio nel medioevo italiano, pp. 361-368.

F. Melis, I vini italiani nel Medioevo, Firenze 1984.

M. Montanari, La fame e l'abbondanza. Storia dell'alimentazione in Europa, Roma-Bari 1997 (ed. or. 1993).

M. Montanari, Tradizioni regionali e modelli culinari. Le materie grasse e l'olio di oliva nella cucina e nell'alimentazione europea, in Olivi e olio nel medioevo italiano, pp. 387-416.

A.M. Nada Patrone, Il consumo del vino nella società pedemontana del tardo medioevo, in Vigne e vini nel Piemonte medievale, pp. 281-299.

I. Naso, Usi alimentari, dietetici e medicinali dell'olio alla fine del medioevo, in Olivi e olio nel medioevo italiano, pp. 417-429.

Olivi e olio nel medioevo italiano, a cura di A. Brugnoli, G.M. Varanini, Bologna 2005.

A. Orlandi, L'olivo e l'olio tra Mediterraneo e Mare del Nord (secoli XIV-XV), in Ars olearia, pp. 107-122.

Francesco Balduccio Pegolotti, La pratica della mercatura, a cura di A. Evans, Cambridge Mass. 1936.

A.I. Pini, Medioevo nel bicchiere: la vite e il vino nella medievistica italiana degli ultimi decen$n i$, in «Quaderni medievali», 29 (1990), pp. 6-38.

A.I. Pini, Il vino del ricco e il vino del povero, in La civiltà del vino, pp. 585-598.

L. Sandri, A.J. Grieco, Appunti per una storia dell'acquavite in Italia: da Taddeo Alderotti alla Fonderia Medicea di Palazzo Pitti (1280-1561), in Grappa e alchimia: un percorso millenario nella storia della distillazione, Roma 1999, pp. 33-48. 


\section{Riccardo Rao}

G.M. Varanini, Lolivicoltura e l'olio gardesano: aspetti della produzione e della commercializzazione dall'VIII al XV secolo, in Olivi e olio nel medioevo italiano, pp. 131-184 (già edito come L'olivicoltura e l'olio gardesano nel Medioevo (aspetti della produzione e della commercializzazione), in Un lago, una civiltà: il Garda, a cura di G. Borelli, Verona 1983).

G.M. Varanini, Le strade del vino. Note sul commercio vinicolo nel tardo Medioevo (con particolare riferimento all'Italia settentrionale), in La civiltà del vino, pp. 635-663.

Vigne e vini nel Piemonte medievale, a cura di R. Comba, Cuneo 1990.

Riccardo Rao

Università degli Studi di Bergamo

riccardo.rao@unibg.it 